Article

\title{
Correlations of the Gastric and Duodenal Microbiota with Histological, Endoscopic, and Symptomatic Gastritis
}

\author{
Hye Seung Han ${ }^{1}$, Sun-Young Lee ${ }^{2, *} \mathbb{C}$, Seo Young $\mathrm{Oh}^{1}{ }^{1}$, Hee Won Moon ${ }^{3}$, Hyunseok Cho ${ }^{4}$ and \\ Ji-Hoon Kim ${ }^{4}$ \\ 1 Department of Pathology, Konkuk University School of Medicine, Seoul 05030, Korea; \\ aphsh@kuh.ac.kr (H.S.H.); syoh@kuh.ac.kr (S.Y.O.) \\ 2 Department of Internal Medicine, Konkuk University School of Medicine, Seoul 05030, Korea \\ 3 Department of Laboratory Medicine, Konkuk University School of Medicine, Seoul 05030, Korea; \\ hanasys@kuh.ac.kr \\ 4 R\&D Center, BioCore. Co. Ltd., Seoul 08511, Korea; hscho@bio-core.com (H.C.); \\ jhkim80@bio-core.com (J.-H.K.) \\ * Correspondence: sunyoung@kuh.ac.kr; Tel.: +82-2-2030-7747; Fax: +82-2-2030-7748
}

Received: 17 January 2019; Accepted: 25 February 2019; Published: 5 March 2019

\begin{abstract}
Mucosal inflammation is characterized by neutrophil and mononuclear cell infiltration. This study aimed to determine the gastric and duodenal microbiota associated with histological, endoscopic, and symptomatic gastritis. Dyspeptic adults who presented for evaluation were included. Subjects with either comorbidities or recent drug intake were excluded. Three endoscopic biopsies were obtained from the antrum, body, and duodenum. Next-generation sequencing for 16S ribosomal RNA V1-V2 hypervariable regions was performed. The correlation between the composition of microbiota and the degree of inflammatory cell infiltration, endoscopic findings, and Patient Assessment of Gastrointestinal Disorders Symptom Severity Index (PAGI-SYM) score was analyzed. In 98 included subjects, microbial communities in the antrum and body showed Bray-Curtis similarity; however, those in the duodenum showed dissimilarity. Histological and endoscopic gastritis was associated with the abundance of Helicobacter pylori and that of commensal bacteria in the stomach. The abundances of Variovorax paradoxus and Porphyromonas gingivalis were correlated with histological gastritis, but not with endoscopic or symptomatic gastritis. The total PAGI-SYM score showed a stronger correlation with the duodenal microbiota (Prevotella nanceiensis and Alloprevotella rava) than with the gastric microbiota (H. pylori, Neisseria elongate, and Corynebacterium segmentosum). Different correlations of the gastric and duodenal microbiota with histological, endoscopic, and symptomatic gastritis were observed for the first time at the species level. H. pylori-negative gastritis is not associated with endoscopic or symptomatic gastritis. Only H. pylori-induced endoscopic gastritis requires gastric cancer surveillance. Owing to the weak correlation with $H$. pylori, symptomatic gastritis should be assessed separately from histological and endoscopic gastritis.
\end{abstract}

Keywords: microbiota; inflammation; endoscopy; stomach; duodenum

\section{Introduction}

Gastritis remains challenging for clinicians, because symptoms may appear even in the absence of histological or endoscopic gastritis. As gastritis-related symptoms are nonspecific, it is thus difficult to detect Helicobacter pylori-infected subjects among the patients with functional dyspepsia [1]. Owing to the lack of difference between H. pylori-induced organic dyspepsia and functional dyspepsia, histopathological and endoscopic findings are more reliable for diagnosing $H$. pylori infection and 
estimating the risk of gastric cancer [2,3]. Furthermore, different clinical, morphological, and serological phenotypes have been reported between $H$. pylori-negative autoimmune gastritis (type A gastritis) and H. pylori-induced antral-dominant type B gastritis [4]. Aside from $H$. pylori, other species may induce gastritis; however, the contribution of other microbiota to symptom generation, mucosal inflammation, and endoscopic findings is still uncertain.

Next-generation sequencing (NGS) analysis of the $16 \mathrm{~S}$ ribosomal RNA (rRNA) hypervariable regions has enabled the understanding of changes in the composition of the gastric microbiota induced by $H$. pylori infection $[5,6]$. Prior to the development of chronic gastritis, acute mucosal inflammation is characterized by simultaneous neutrophil and mononuclear cell infiltration [2,7]. Acute inflammation is often found when the mucosal microbiota is dominated by the pathogen such as $H$. pylori [5,6,8-13]. $H$. pylori affects adjacent organs, and is more abundant in the duodenal lumen than in the duodenal mucosa [14]. Because duodenal dysfunction and abnormal responses may also induce functional dyspepsia [15], the composition of the duodenal microbiota may differ between the symptomatic and asymptomatic subjects. Although recent advances in NGS analysis have enabled the identification of the composition of microbiota in the duodenal mucosa, microbiota other than H. pylori that contribute to mucosal inflammation, carcinogenesis, or symptom generation remain unknown.

A pathogenic species may induce mucosal inflammation, specific endoscopic findings, and gastrointestinal symptoms. Conversely, the microbiota that plays a protective role against inflammation might be underrepresented in the inflamed mucosa. In this study, we attempted to determine the composition of gastric and duodenal microbiota according to the degree of inflammatory cell infiltration and the presence of endoscopic gastritis. To understand the differences among histological, endoscopic, and symptomatic gastritis, we also analyzed the correlation between the composition of microbiota and gastrointestinal symptoms.

\section{Materials and Methods}

\subsection{Study Subjects}

Korean adults who visited our Digestive Disease Center for the evaluation of dyspeptic symptom were enrolled in a prospective setting. Subjects with either comorbidities or recent drug intake within the last 3 months (including antimicrobials, acid suppressants, antidepressants, antithrombotic agents, laxatives, lipid-lowering agents, hypoglycemic agents, probiotics, hormone replacement therapy drugs, and traditional Chinese medicine) were excluded. Ninety-eight subjects (mean age, $37.9 \pm 11.9$ years; 21 male and 77 female) provided signed informed consents prior to the procedure, and their details were omitted to ensure anonymity. None of the included subjects were vegetarians.

Ethics approval: This study was approved by the institutional review board of Konkuk University Medical Center which confirmed that the study followed the Declaration of Helsinki. This study is registered as KCT0000718 at the Clinical Research Information Service (https:/ / cris.nih.go.kr).

\subsection{Upper Gastrointestinal Endoscopy and Biopsy Procedures}

Before endoscopic examination, subjects were asked to score their symptoms from 0 (none) to 5 (very severe) on the 20-item Patient Assessment of Gastrointestinal Disorders Symptom Severity Index (PAGI-SYM) questionnaire, as described previously [16]. Endoscopic procedures were performed by one gastroenterologist (Dr. S.-Y. Lee) using the GIF-Q260 endoscope (Olympus Co., Ltd., Tokyo, Japan). Three biopsies were obtained from the greater curvature sides of the mid-antrum and mid-body, in addition to the descending duodenum. To minimize the risk of bile contamination, duodenal biopsy specimens were obtained from the opposite side of the ampulla of Vater. Specimens were taken using $2.8 \mathrm{~mm}$ biopsy forceps (FB-230K or FB-24K-1, Olympus Co, Ltd., Tokyo, Japan).

Endoscopic findings were described according to the updated Sydney classification system in addition to $H$. pylori-related endoscopic findings $[2-4,17,18]$. The presence of the following was recorded: (1) multiple minute hemorrhagic spots in the fundus, (2) hypertrophic gastric rugae 
measuring over $5 \mathrm{~mm}$, (3) mucosal nodularity, including small granular-type nodular gastritis (chicken skin-like mucosa showing multiple submucosal nodules measuring 1-2 $\mathrm{mm}$ in size), large nodular-type nodular gastritis (multiple submucosal nodules measuring $3-4 \mathrm{~mm}$ in size), and metaplastic gastritis (irregular whitish elevations and/or depressed patchy erythema), (4) advanced gastric atrophy (visible submucosal vessels extending up to the body from the antrum), (5) erosive gastritis (raised, regular-sized, hyperemic erosions), (6) chronic superficial gastritis (regular linear hyperemic streaks), (7) gastric xanthoma (yellowish plaque), and (8) hematin deposits (intramural hemorrhage). Other notable changes in the background mucosa were recorded.

\subsection{Histopathological Assessment}

Gastric and duodenal biopsy specimens were evaluated by one pathologist (Dr. H. S. Han) blinded to the subjects' clinical information and status. All the biopsied specimens were fixed with $10 \%$ neutral-buffered formalin and were embedded in paraffin blocks. After hematoxylin-eosin (H\&E) and Giemsa staining, microscopic findings were described. The specimens were observed with the aid of microscope (Olympus BX51, Olympus Co, Ltd., Tokyo, Japan). Degrees of neutrophil and mononuclear cell infiltration, atrophy, and intestinal metaplasia were scored from 0 to 3 (no, mild, moderate, and marked degrees) according to the updated Sydney classification system (Figure S1).

\subsection{Library Preparation for $16 S$ rRNA Gene Sequencing}

The 16S rRNA V1-V2 hypervariable regions were analyzed based on recent study findings on the gastric and/or duodenal mucosal microbiota using endoscopic biopsied specimens $[14,19,20]$. To prepare the library for NGS analysis of the 16S rRNA V1-V2 hypervariable regions, DNA extraction was conducted for specimens obtained from the antrum, body, and duodenum, as described [21]. During DNA extraction, proteinase K (Takara Bio. Inc., Shiga, Japan) was used to effectively break down the thick bacterial cell wall, and incubation for cell lysis was performed at $100^{\circ} \mathrm{C}$. All isolated samples were checked for quantity and quality prior to polymerase chain reaction (PCR) amplification.

Bacterial 16S rRNA V1-V2 hypervariable regions were amplified using primers (Meta-8F-V1, V2: AGAGTTTGATCMTGGCTCAG and Meta-338R-V1, V2: GCTGCCTCCCGTAGGAGT) [22]. PCR amplification of the V1-V2 hypervariable regions was performed in a thermal controller (TP600, Takara Bio Inc., Shiga, Japan) using $12 \mu \mathrm{L}$ of DNA extract. The final PCR reaction mix volume (30 $\mu \mathrm{L})$ was achieved by adding $15 \mu \mathrm{L}$ of BioFact $2 \times$ multistar master mix (Biofact Co., Ltd., Daejeon, Korea) and $1.5 \mu \mathrm{L}$ (10 pmole) of each primer. The initial denaturation was performed at $95^{\circ} \mathrm{C}$ for $10 \mathrm{~min}$. Thereafter, 40 cycles of denaturation at $95^{\circ} \mathrm{C}$ for $30 \mathrm{~s}$, annealing at $58^{\circ} \mathrm{C}$ for $40 \mathrm{~s}$, and extension at $72{ }^{\circ} \mathrm{C}$ for $5 \mathrm{~min}$ were carried out. The final extension was performed at $72{ }^{\circ} \mathrm{C}$ for $7 \mathrm{~min}$. A negative control without template was included in PCR products.

End-repair, ligation, nick-repair, and final amplification processes for sequencing libraries were performed using Ion Plus Fragment Library Kit and Ion Xpress Barcode Adapters (Thermo Fisher Scientific Inc., Waltham, MA, USA) according to the manufacturer's protocol. The 16S rRNA libraries were purified using Agencourt AMPure XP reagent (Beckman Coulter, Brea, CA, USA). The respective size of each library and the absence of contaminants were verified using high-sensitivity DNA Kit on Agilent 2100 Bioanalyzer (Agilent Technologies, Santa Clara, CA, USA).

\subsection{Template Sequencing and Data Availability}

Template preparation and sequencing were performed with the aid of Ion Chef System and Ion S5 XL System with Ion 530 Chip Kit (Thermo Fisher Scientific Inc., Waltham, MA, USA). The FASTQ data were generated using the Torrent Suite ${ }^{\mathrm{TM}}$ software version 5.8 (Thermo Fisher Scientific Inc., Waltham, MA, USA) by sequencing the V1-V2 hypervariable regions of $16 \mathrm{~S}$ rRNA gene. The FASTQ sequence files are available in the National Center for Biotechnology Information (NCBI) Bioproject under the accession number of PRJNA486978. 


\subsection{Quality Control of Sequence Data}

The FASTQ data were further analyzed for quality control. After obtaining the sequences from each sample, trimmed reads were mapped. The final files were generated by demultiplexing the data and removing the primer. Reliable data were obtained by excluding Phred quality scores below 20, polyclonal reads with low quality, and 3 '-end adaptors of reads.

\subsection{Bioinformatics Processing and Sequence Analysis}

Ion Reporter ${ }^{\mathrm{TM}}$ software version 5.6 (Thermo Fisher Scientific Inc., Waltham, MA, USA) was used to process the FASTQ files. Read sequences were checked for a minimum read length of $150 \mathrm{bp}$. Data filtering was performed by removing low copy numbers (threshold $<10$ ) and reads with an alignment coverage of $<90 \%$. Based on Ion Reporter Metagenomics $16 \mathrm{~S}$ algorithms, automatic analyses were conducted using the metagenomics workflow in the Ion Reporter software. The linked Quantitative Insights into Microbial Ecology (QIIME) workflow on the Ion Reporter Server processed the FASTQ files as input data, and reported operational taxonomic unit (OTU) tables as output data.

\subsection{Screening and Assessment for Organisms}

The sequence reads were aligned against the reference sequences exhibited in Greengenes version 13.5 (Greengenes Database Consortium) and MicroSEQ 16S rRNA Reference Library version 2013.1 (Thermo Fisher Scientific Inc., Waltham, MA, USA). With respect to taxonomy assignment, OTUs with $\geq 10$ copy numbers were taxonomically classified using two databases. The reads were matched with clean OTUs showing a sequence similarity of $>97 \%$ for the entire length, as described previously [23].

\subsection{Assessment of Alpha and Beta Diversity Indices}

To minimize the effects of uneven sampling, diversity indices were calculated after normalizing the read numbers in each sample. Alpha diversity was exhibited as Chao1, Shannon, and Simpson indices using the linked QIIME workflow. To determine beta diversity, transformed OTU counts were used for principal coordinate analysis (PCoA). A PCoA plot based on the Bray-Curtis dissimilarity was constructed using the linked QIIME workflow.

\subsection{Statistical Analysis}

For the comparison of PCoA plots, permutational multivariate analysis of variance test was performed using "vegan" R package version 2.2-1. For continuous variables, means and standard deviations were provided using the $t$-test. For continuous variables with asymmetrical distribution, median values and ranges were provided using the Kruskal-Wallis test. Categorical variables were described as frequencies using chi-square test or Fisher's exact test. Analysis was performed using PASW version 17.0 (SPSS Inc., Chicago, IL, USA). A $p$-value of $<0.05$ was considered statistically significant.

For the comparison between the $H$. pylori-infected and non-infected subjects, $>1 \% H$. pylori relative abundance was defined as H. pylori infection [24]. For multiple comparisons, continuous variables were analyzed by using the analysis of variance (ANOVA) with Bonferroni correction. Chi-squared test with Bonferroni correction was used for categorical variables. Correlation analysis was performed to verify the relationship between the relative abundance of microbiota and the degree of inflammatory cell infiltration, and described as correlation coefficient values $(r)$ with a $p$-value. Furthermore, correlation analysis was performed to evaluate the association between the relative abundance of microbiota and PAGI-SYM scores. To exclude correlations found by chance, statistical significance was set at $p<0.0083$ $(p<0.05$ divided by six symptom subscales) after multiple testing correction. 


\section{Results}

\subsection{Different Compositions Between the Gastric and Duodenal Microbiota}

A total of 573 different species (10 phyla, 209 families, and 335 genus) were detected in the stomach in 98 subjects. In the duodenum, 500 different species (9 phyla, 183 families, and 260 genera) were found. Beta diversity indicated a significant similarity between the antrum and the body (Figure 1). Common species found in the antrum, body, and duodenum are summarized in Table S1. Differences between men and women were found only in the relative abundance of Prevotella pallens $(p=0.009)$ and Streptococcus sp. $(p=0.007)$ in the stomach. The median relative abundance of $P$. pallens was higher in women $(0.06 \%$; range, $0-8 \%$ ) than in men $(0.02 \%$; range, $0-11 \%)$. The median relative abundance of Streptococcus sp. was higher in men $(0.09 \%$; range, $0-6 \%)$ than in women $(0.04 \%$; range, $0-2 \%)$.

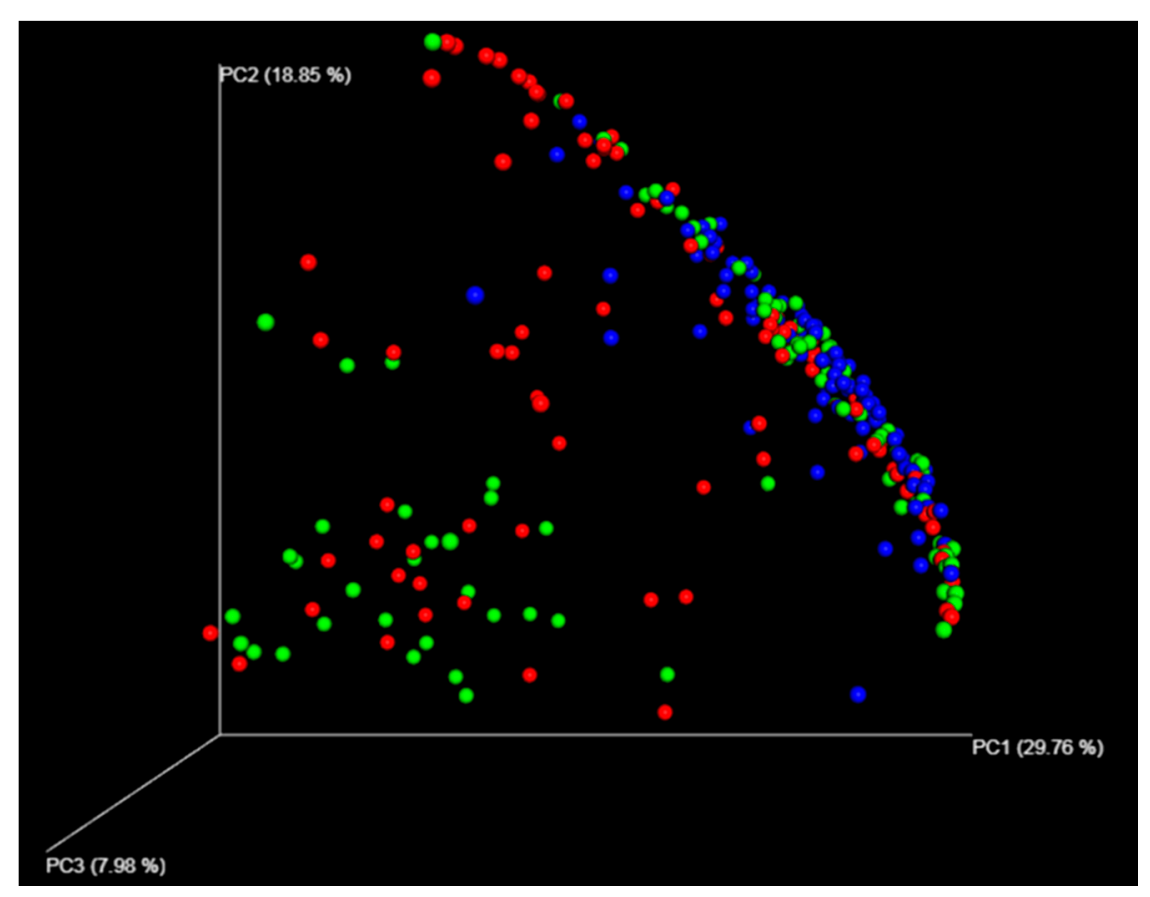

Figure 1. Beta diversity comparisons of microbial communities in the antrum, body, and duodenum. Principal coordinate analysis (PCoA) plots for the antrum (red), body (green), and duodenum (blue) are shown to determine Bray-Curtis distances. In the permutational multivariate analysis of variance, microbial communities in the antrum and the body showed similarity with a pseudo F-value of 4.52 $(p=0.003, q=0.0030)$, whereas those in the antrum and duodenum showed dissimilarity with a pseudo F-value of 16.15 ( $p=0.001, q=0.0015)$. Furthermore, microbial communities in the body and duodenum showed Bray-Curtis dissimilarity with a pseudo F-value of $11.86(p=0.001, q=0.0015)$.

With respect to the relative abundance of species from different biopsy sites, the strongest correlation was observed between $H$. pylori in the antrum and H. pylori in the body $(r=0.904, p<0.001)$. The correlation coefficient values for the relative abundance of $H$. pylori in the duodenum and $H$. pylori in the antrum and body were $0.064(p=0.528)$ and $0.260(p=0.010)$, respectively. Regardless of the presence of $H$. pylori infection, Brevundimonas aurantiaca was dominant in the duodenum in 89 (90.8\%) subjects. Only 14 subjects showed $H$. pylori in the duodenum among the $31 \mathrm{H}$. pylori-infected subjects. H. pylori-infected subjects showed lower diversity indices in the stomach, and higher diversity indices in the duodenum than non-infected subjects (Table 1). 
Table 1. Differences between subjects according to the status of Helicobacter pylori infection.

\begin{tabular}{|c|c|c|c|}
\hline Findings & $\begin{array}{c}\text { With no Helicobacter } \\
\text { pylori Infection }(n=61)\end{array}$ & $\begin{array}{l}\text { With H. pylori Infection } \\
\qquad(n=31)\end{array}$ & $\begin{array}{l}\text { With past H. pylori } \\
\text { Infection }(n=6)\end{array}$ \\
\hline Age (year-old) & $35.4 \pm 11.9$ & $40.5 \pm 9.9$ & $49.8 \pm 13.9^{*, * *}$ \\
\hline Sex (male:female) & $16: 45$ & $3: 28$ & $2: 4$ \\
\hline $\begin{array}{l}\text { Body mass index } \\
\qquad\left(\mathrm{kg} / \mathrm{m}^{2}\right)\end{array}$ & $21.9 \pm 3.4$ & $22.4 \pm 3.8$ & $21.7 \pm 2.2$ \\
\hline \multicolumn{4}{|c|}{ Antrum: $16 \mathrm{~S}$ ribosomal RNA (rRNA) sequencing analysis findings } \\
\hline Target reads & $20,759 \pm 18373$ & $18,744 \pm 11,508$ & $26,175 \pm 9597$ \\
\hline Chao 1 index & $46.9 \pm 16.6$ & $46.7 \pm 12.6$ & $60.2 \pm 9.4$ \\
\hline Shannon diversity index & $3.7 \pm 0.5$ & $3.4 \pm 0.7 *$ & $3.9 \pm 0.3^{* *}$ \\
\hline Simpson diversity index & $0.85 \pm 0.05$ & $0.78 \pm 0.12 *$ & $0.86 \pm 0.04 * *$ \\
\hline \multicolumn{4}{|c|}{ Body: 16S rRNA sequencing analysis findings } \\
\hline Target reads & $14,998 \pm 9734$ & $13,041 \pm 7148$ & $12,271 \pm 9245$ \\
\hline Chao 1 index & $39.2 \pm 7.4$ & $32.0 \pm 8.9 *$ & $33.8 \pm 12.1$ \\
\hline Shannon diversity index & $3.4 \pm 0.4$ & $2.6 \pm 0.6 *$ & $3.3 \pm 0.7^{* *}$ \\
\hline Simpson diversity index & $0.82 \pm 0.04$ & $0.68 \pm 0.13 *$ & $0.82 \pm 0.06^{* *}$ \\
\hline \multicolumn{4}{|c|}{ Duodenum: 16S rRNA sequencing analysis findings } \\
\hline Target reads & $9329 \pm 4067$ & $11,440 \pm 6949$ & $9834 \pm 2278$ \\
\hline Chao 1 index & $31.5 \pm 6.2$ & $37.0 \pm 7.2 *$ & $32.8 \pm 2.7$ \\
\hline Shannon diversity index & $3.2 \pm 0.3$ & $3.5 \pm 0.4 *$ & $3.3 \pm 0.2$ \\
\hline Simpson diversity index & $0.81 \pm 0.05$ & $0.83 \pm 0.05$ & $0.83 \pm 0.02$ \\
\hline \multicolumn{4}{|c|}{ Antrum: updated Sydney system (no:mild:moderate:marked) } \\
\hline Neutrophil & $4: 53: 4: 0$ & $0: 5: 20: 6^{*}$ & $0: 6: 0: 0 * *$ \\
\hline Mononuclear cell & $58: 2: 0: 1$ & $6: 10: 12: 3$ * & $6: 0: 0: 0 * *$ \\
\hline Atrophy & $37: 24: 0: 0$ & $15: 16: 0: 0$ & $3: 3: 0: 0$ \\
\hline Intestinal metaplasia & $60: 1: 0: 0$ & $28: 2: 1: 0$ & $5: 1: 0: 0$ \\
\hline \multicolumn{4}{|c|}{ Body: updated Sydney system (no:mild:moderate:marked) } \\
\hline Neutrophil & 1:58:1:1 & $0: 8: 20: 3$ * & $0: 6: 0: 0 * *$ \\
\hline Mononuclear cell & 59:0:1:1 & $6: 6: 18: 1 *$ & $6: 0: 0: 0 * *$ \\
\hline Atrophy & $35: 26: 0: 0$ & 23:8:0:0 & $4: 2: 0: 0$ \\
\hline Intestinal metaplasia & 67:0:0:0 & $30: 1: 0: 0$ & 6:0:0:0 \\
\hline \multicolumn{4}{|c|}{ Duodenum: inflammatory cell infiltration (no:mild) } \\
\hline Neutrophil & $55: 6$ & $22: 8$ & $4: 2$ \\
\hline Mononuclear cell & 9:52 & $4: 27$ & $0: 6$ \\
\hline
\end{tabular}

For continuous variables, analysis of variance (ANOVA) with Bonferroni correction was used. For categorical variables, chi-squared test with Bonferroni correction was used. * Significantly different with 61 subjects with no H. pylori infection. ${ }^{* *}$ Significantly different with 31 subjects with $H$. pylori infection.

\subsection{Gastric and Duodenal Microbiota Related to Mucosal Inflammation}

The composition of the gastric microbiota differed according to the combined inflammation score (Figure 2A). The combined inflammation score was correlated with the relative abundance of three species in the stomach (H. pylori, Variovorax paradoxus, and Porphyromonas gingivalis) and two species in the duodenum (H. pylori and Leptotrichia genomosp).

The relative abundance of Propionibacterium acnes $(r=-0.475)$, Pseudomonas veronii $(r=-0.349)$, Pseudomonas sp. $(r=-0.345)$, Dechloromonas sp. $(r=-0.291)$, Staphylococcus epidermidis $(r=-0.272)$, Cloacibacterium rupense ( $r=-0.267)$, Escherichia coli $(r=-0.265)$, Hydrogenophilus hirschi, $(r=-0.227)$, and Bacillus sp. $(r=-0.225)$ was negatively associated with the combined inflammation score in the stomach. In the duodenum, the relative abundance of Moraxella osloensis $(r=-0.297)$, S. epidermidis $(r=-0.245)$, and Actinomyces odontolyticus $(r=-0.217)$ was negatively correlated with inflammation. 
Gastric and duodenal microbiota that showed significant correlation with inflammatory cell infiltration at the genus or species level are summarized in Table 2.

\section{Combined score of neutrophil and mononuclear cell infiltration}

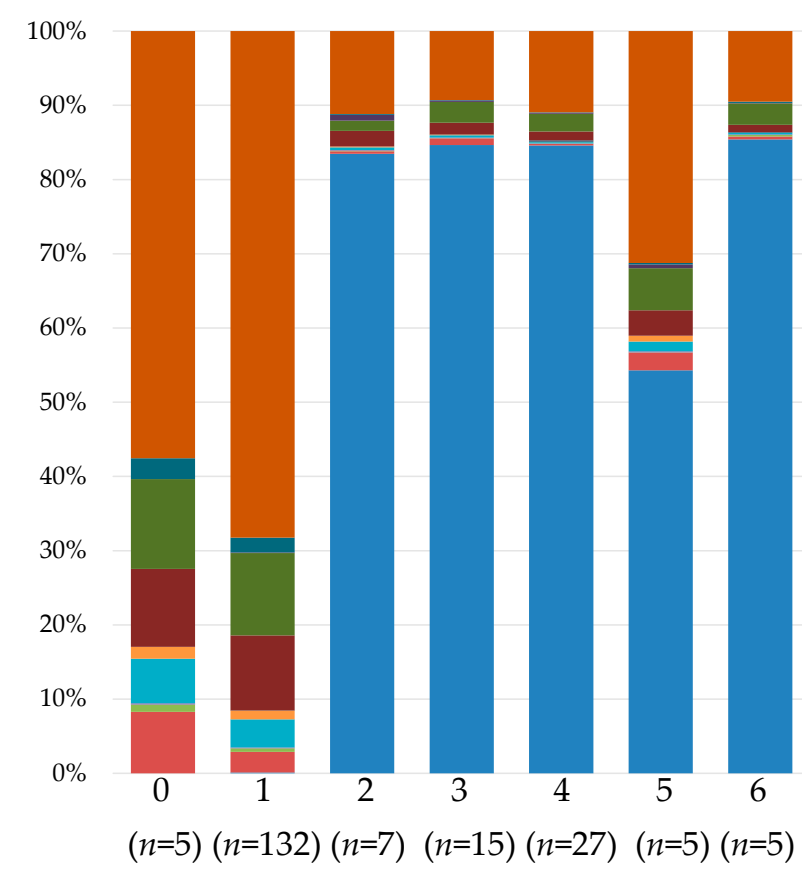

A $\quad \begin{gathered}\text { All subjects } \\ \text { (98 antrum \& } 98 \text { body) }\end{gathered}$

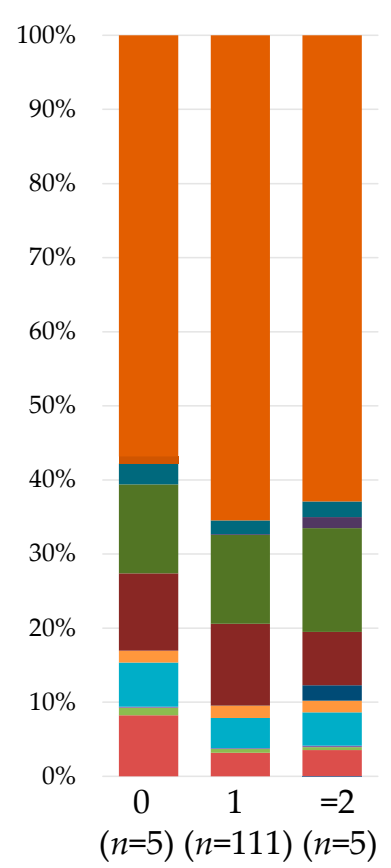

B H. pylori-negative subjects (61 antrum \& 61 body)

Figure 2. Composition of the gastric microbiota according to the combined inflammation score of neutrophil and mononuclear cell infiltration. (A) The composition of the gastric microbiota is shown according to the combined inflammation score. Scores of $0,1,2$, and 3 indicate no, mild, moderate, and marked degrees, respectively, of neutrophil or mononuclear cell infiltration. The composition of gastric microbiota differed between the subjects with a score $<2$ and those with a score $\geq 2$. (B) Among the $61 \mathrm{H}$. pylori-negative subjects, only five subjects had a combined inflammation score $\geq 2$. Significant differences were found with the relative abundance of Variovorax paradoxus and Porphyromonas gingivalis between the subjects with scores $\geq 2$ and those with scores $<2$. S. epidermidis: Staphylococcus epidermidis; C. rupense: Cloacibacterium rupense; P. acnes: Propionibacterium acnés; P. veronii: Pseudomonas veronii; H. hirschii; Hydrogenophilus hirschii; E. coli: Escherichia coli. 
Table 2. Significant correlations between the relative abundance of microbiota and the combined inflammation score.

\begin{tabular}{|c|c|c|c|}
\hline \multicolumn{2}{|c|}{ Microbiota } & \multicolumn{2}{|c|}{ Combined Inflammation Score } \\
\hline Site & Level & Positive Correlation & Negative Correlation \\
\hline \multirow[b]{2}{*}{ Antrum } & Genus & Helicobacter 0.794 & $\begin{array}{c}\text { Dechloromonas }-0.354 \\
\text { Staphylococcus }-0.326 \\
\text { Pseudomonas }-0.294\end{array}$ \\
\hline & Species & Helicobacter pylori 0.800 & $\begin{array}{c}\text { Propionibacterium acnes }-0.445 \\
\text { Pseudomonas veronii }-0.360 \\
\text { Dechloromonas sp. }-0.323 \\
\text { Cloacibacterium rupense }-0.320 \\
\text { Pseudomonas sp. }-0.320 \\
\text { Staphylococcus epidermidis }-0.280 \\
\text { Hydrogenophilus hirschii }-0.250 \\
\text { Streptococcus sp. }-0.231\end{array}$ \\
\hline \multirow[t]{2}{*}{ Body } & Genus & $\begin{array}{c}\text { Helicobacter } 0.713 \\
\text { Variovorax } 0.324\end{array}$ & $\begin{array}{c}\text { Propionibacterium }-0.469 \\
\text { Corynebacterium }-0.457 \\
\text { Methylobacterium }-0.454 \\
\text { Pseudomonas }-0.402 \\
\text { Escherichia }-0.342 \\
\text { Hydrogenophilus }-0.307 \\
\text { Prevotella }-0.307 \\
\text { Staphylococcus }-0.280 \\
\text { Sphingomonas }-0.258 \\
\text { Dechloromonas sp. }-0.256\end{array}$ \\
\hline & Species & $\begin{array}{c}\text { Helicobacter pylori } 0.719 \\
\text { Variovorax paradoxus } 0.325 \\
\text { Porphyromonas gingivalis } 0.249\end{array}$ & $\begin{array}{c}\text { Propionibacterium acnes }-0.514 \\
\text { Pseudomonas sp. }-0.377 \\
\text { Pseudomonas veronii }-0.371 \\
\text { Escherichia coli }-0.331 \\
\text { Dechloromonas sp. }-0.279 \\
\text { Bacillus sp. }-0.274 \\
\text { Staphylococcus epidermidis }-0.265\end{array}$ \\
\hline \multirow[b]{2}{*}{ Duodenum } & Genus & Helicobacter 0.215 & - \\
\hline & Species & $\begin{array}{l}\text { Leptotrichia genomosp. } 0.218 \\
\text { Helicobacter pylori } 0.201\end{array}$ & $\begin{array}{c}\text { Moraxella osloensis }-0.279 \\
\text { Staphylococcus epidermidis }-0.245 \\
\text { Actinomyces odontolyticus }-0.217\end{array}$ \\
\hline
\end{tabular}

Pearson's correlation coefficient $(r)$ is shown for each microbiota.

\subsection{Microbiota Associated with Abnormal Endoscopic Findings}

H. pylori was more abundant in subjects with hemorrhagic spots $(n=6)$, hypertrophic rugae $(n=7)$, advanced atrophy $(n=9)$, and mucosal nodularity $(n=13)$ than in their counterparts (Figure 3$)$. No correlation with the composition of microbiota was observed in subjects with other endoscopic findings (raised hyperemic erosions $(n=3)$, hematin deposits $(n=3)$, linear hyperemic streaks $(n=2)$, and gastric xanthoma $(n=1))$. P. acnes, P. veronii, Pseudomonas sp., S. epidermidis, and C. rupense were abundant in the presence of regular arrangement of collecting venules, which indicates intact gastric mucosa without endoscopic gastritis (Table S2). All species related to endoscopic gastritis (H. pylori, P. acnes, P. veronii, Pseudomonas sp., S. epidermidis, and C. rupense) were found among the species associated with histological gastritis. 


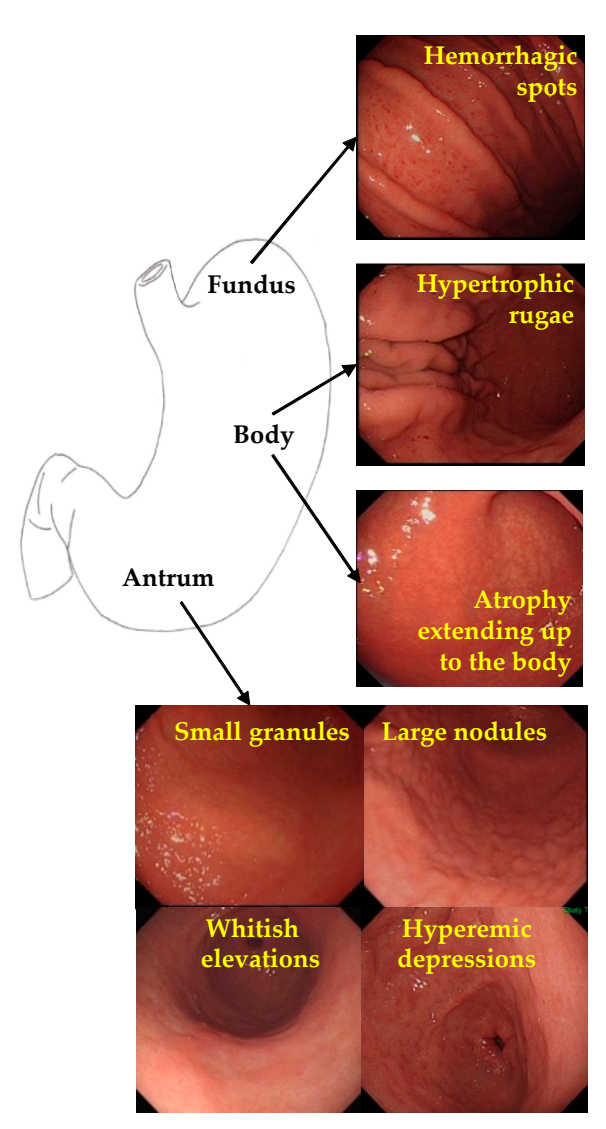

\begin{tabular}{|c|c|c|c|c|}
\hline \multicolumn{4}{|c|}{$\begin{array}{l}\text { Relative abundance (\%) of species related to the presence of } \\
\text { multiple tiny hemorrhagic spots in the fundus }\end{array}$} & \multirow[t]{2}{*}{$p$-value } \\
\hline & cies & Present $(n=6)$ & Absent $(n=92)$ & \\
\hline Antrum & H. pylori & $76.2(45.9 \sim 93.9)$ & $0.3(0 \sim 93.6)$ & $<0.001$ \\
\hline Body & $\begin{array}{l}\text { H. pylori } \\
\text { P. veronii } \\
\text { P. acnes }\end{array}$ & $\begin{array}{c}90.8(76.2 \sim 95.2) \\
0.2(0 \sim 1.5) \\
1.0(0 \sim 3.1)\end{array}$ & $\begin{array}{l}0.6(0 \sim 98.4) \\
3.3(0 \sim 25.1) \\
8.2(0 \sim 29.1)\end{array}$ & $\begin{array}{c}<0.001 \\
0.038 \\
0.007\end{array}$ \\
\hline
\end{tabular}

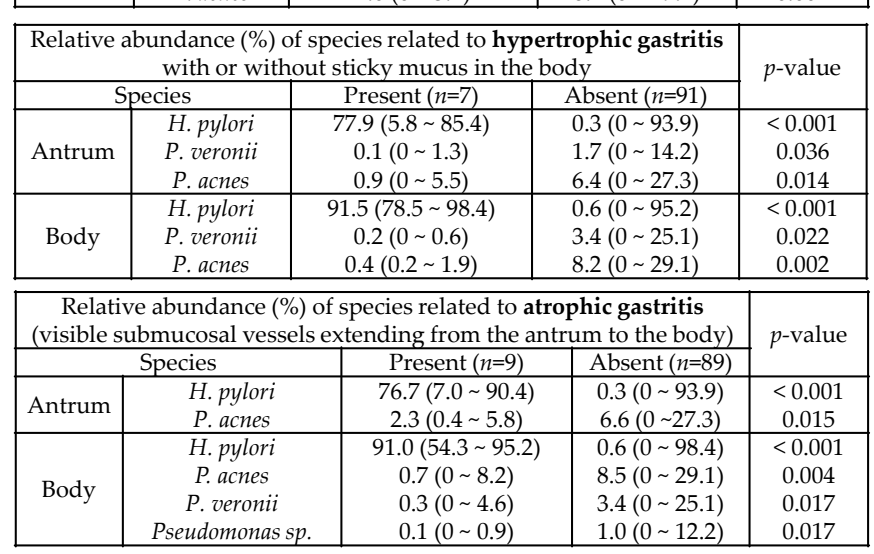

\begin{tabular}{|c|c|c|c|c|}
\hline \multicolumn{4}{|c|}{$\begin{array}{l}\text { Relative abundance }(\%) \text { of species related to diffuse mucosal } \\
\text { nodularity including nodular gastritis and metaplastic gastritis }\end{array}$} & \multirow[t]{2}{*}{$p$-value } \\
\hline & Species & Present $(n=13)$ & Absent $(n=85)$ & \\
\hline \multirow{4}{*}{ Antrum } & H. pylori & $76.7(7.0 \sim 91.2)$ & $0.2(0 \sim 93.9)$ & $<0.001$ \\
\hline & P. veronii & $0.3(0 \sim 3.3)$ & $1.7(0 \sim 14.2)$ & 0.025 \\
\hline & P. acnes & $2.0(0 \sim 6.6)$ & $7.4(0 \sim 27.3)$ & 0.006 \\
\hline & C. rupense & $4.7(1.3 \sim 20.8)$ & $12.7(0 \sim 76.5)$ & 0.028 \\
\hline \multirow{6}{*}{ Body } & H. pylori & $90.5(54.3 \sim 95.2)$ & $0.5(0 \sim 98.4)$ & $<0.001$ \\
\hline & P. veronii & $0.4(0 \sim 4.6)$ & $3.8(0 \sim 25.1)$ & 0.003 \\
\hline & Pseudomonas sp. & $0.1(0 \sim 1.0)$ & $1.1(0 \sim 12.2)$ & 0.007 \\
\hline & P. acnes & $1.7(0.3 \sim 8.2)$ & $9.5(0 \sim 29.1)$ & $<0.001$ \\
\hline & C. rupense & $0.8(0 \sim 5.1)$ & $5.8(0 \sim 66.4)$ & 0.014 \\
\hline & S. epidermidis & $0.1(0 \sim 1.6)$ & $1.6(0 \sim 26.8)$ & 0.028 \\
\hline
\end{tabular}

Figure 3. Significant endoscopic findings that were correlated with the relative abundance of microbiota. Helicobacter pylori was abundant in the presence of hemorrhagic spots, hypertrophic rugae, advanced atrophy, and mucosal nodularity, whereas Pseudomonas veronii and Propionibacterium acnes were abundant in the absence of these findings. Pseudomonas sp. was abundant in the absence of atrophy and nodularity. Moreover, Cloacibacterium rupense and Staphylococcus epidermidis were abundant in the absence of nodularity. The median relative abundance of each species were provided with range (minimum-maximum) using the Kruskal-Wallis test. H. pylori: Helicobacter pylori; P. veronii: Pseudomonas veronii; P. acnes: Propionibacterium acnés; C. rupense: Cloacibacterium rupense; S. epidermidis: Staphylococcus epidermidis.

\subsection{Microbiota Associated with the PAGI-SYM Score}

The total PAGI-SYM score showed positive correlations with Prevotella nanceiensis $(r=0.273)$ and Alloprevotella rava $(r=0.209)$ in the duodenum. Stronger correlations were found with the duodenal microbiota than the gastric microbiota (H. pylori, $r=0.165$; Neisseria elongata, $r=0.143$; Corynebacterium segmentosum, $r=0.143$; $P$. pallens, $r=-0.196$; P. acnes, $r=-0.171$; S. epidermidis, $r=-0.145)$. Correlation with the duodenal microbiota was mostly observed with bloating, nausea, vomiting, and lower abdominal pain (Table 3). 
Table 3. Gastric and duodenal microbiota correlated with the Patient Assessment of Gastrointestinal Disorders Symptom Severity Index (PAGI-SYM) scores.

\begin{tabular}{|c|c|c|c|}
\hline \multirow{2}{*}{$\begin{array}{c}\text { PAGI-SYM } \\
\text { Questionnaires }\end{array}$} & \multirow{2}{*}{ Site } & \multicolumn{2}{|c|}{ Correlation with the Relative Abundance of Species } \\
\hline & & Positive Correlation & Negative Correlation \\
\hline $\begin{array}{l}\text { Heartburn and } \\
\text { regurgitation }\end{array}$ & Stomach & Corynebacterium segmentosum 0.189 & Prevotella pallens - 0.193 \\
\hline \multirow{3}{*}{ Nausea and vomiting } & Duodenum & Prevotella nanceiensis 0.283 & $\begin{array}{l}\text { Propionibacterium acnes }-0.220 \\
\quad \text { Prevotella pallens }-0.215\end{array}$ \\
\hline & $\begin{array}{l}\text { Stomach } \\
\text { Duodenum }\end{array}$ & $\begin{array}{c}\text { Helicobacter pylori } 0.239 \\
\text { Prevotella nanceiensis } 0.302 \\
\text { Actinomyces odontolyticus } 0.265\end{array}$ & $\begin{array}{l}- \\
-\end{array}$ \\
\hline & Stomach & H. pylori 0.194 & $\begin{array}{c}\text { Propionibacterium acnes }-0.234 \\
\text { Staphylococcus epidermidis }-0.145\end{array}$ \\
\hline \multirow{2}{*}{ Upper abdominal pain } & Stomach & - & - \\
\hline & Duodenum & - & - \\
\hline \multirow{2}{*}{$\begin{array}{l}\text { Fullness and early satiety } \\
\text { Lower abdominal pain }\end{array}$} & Stomach & 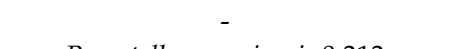 & Dechloromonas sp. -0.189 \\
\hline & $\begin{array}{l}\text { Duodenum } \\
\text { Stomach }\end{array}$ & $\begin{array}{c}\text { Prevotella nanceiensis } 0.313 \\
\text { H. pylori } 0.228\end{array}$ & -1 \\
\hline \multirow{2}{*}{$\begin{array}{c}\text { PAGI-SYM } \\
\text { Questionnaires }\end{array}$} & \multirow{2}{*}{ Site } & \multicolumn{2}{|c|}{ Correlations Found in 61 H. pylori-Negative Subjects } \\
\hline & & Positive Correlation & Negative Correlation \\
\hline $\begin{array}{l}\text { Heartburn \& } \\
\text { regurgitation }\end{array}$ & Stomach & Corynebacterium segmentosum 0.274 & - \\
\hline Bloating & Duodenum & $\begin{array}{l}\text { Pseudomonas grimontii } 0.308 \\
\text { Cloacibacterium normanense } 0.281\end{array}$ & - \\
\hline Nausea and vomiting & $\begin{array}{l}\text { Duodenum } \\
\text { Stomach }\end{array}$ & $\begin{array}{c}\text { Actinomyces odontolyticus } 0.348 \\
\text { Prevotella nanceiensis } 0.258 \\
\text { Neisseria enlongata } 0.245 \\
\text { Actinobacillus parahaemolyticus }\end{array}$ & - \\
\hline Upper abdominal pain & Duodenum & $\begin{array}{c}0.364 \\
\text { Rothia mucilaginosa } 0.246 \\
\text { Pseudomonas grimontii } 0.242\end{array}$ & $\begin{array}{c}\text { Paracoccus sp. }-0.242 \\
\text { Neisseria perflava }-0.242\end{array}$ \\
\hline \multirow{2}{*}{ Fullness and early satiety } & Duodenum & Porphyromonas catoniae 0.332 & - \\
\hline & Stomach & Pantoea sp. 0.278 & - \\
\hline Lower abdominal pain & Stomach & Neisseria enlongata 0.249 & - \\
\hline
\end{tabular}

\subsection{Association Between the Microbiota Correlated with Histological, Endoscopic, and Symptomatic Gastritis}

In the presence of hemorrhagic spots on endoscopic examination, the severity scores of heartburn and regurgitation $(p=0.004)$, bloating $(p=0.001)$, and lower abdominal pain $(p=0.001)$ were significantly higher than those without hemorrhagic spots (Table S3). Other endoscopic findings were not associated with symptom subscale severity.

\subsection{Microbiota Associated with Gastritis in H. pylori-Negative Subjects}

In $61 \mathrm{H}$. pylori-negative subjects, the combined inflammation score was correlated with the relative abundances of $V$. paradoxus $(r=0.670)$ and $P$. gingivalis $(r=0.259)$ in the stomach (Figure 2B). None of the gastric microbiota showed an inverse correlation with the combined score. In the duodenum, the combined inflammation score was inversely correlated with the abundances of S. epidermidis $(r=-0.346)$ and M. osloensis $(r=-0.305)$. No duodenal microbiota showed a positive correlation with the combined inflammation score.

Only eight subjects had specific endoscopic findings (hyperemic raised erosions in 3 subjects, hematin deposits in 3 subjects, and linear hyperemic streaks in 2 subjects). No significant correlation was found between the presence of these endoscopic findings and the composition of the microbiota.

The total PAGI-SYM score was correlated with the abundance of $N$. elongata $(r=0.207)$, C. segmentosum $(r=0.235)$, P. pallens $(r=-0.237)$ in the stomach and that $A$. odontolyticus $(r=0.243)$, Pseudomonas grimontii $(r=0.238)$, and Paracoccus sp. $(r=-0.207)$ in the duodenum. Stronger positive correlations were found with the duodenal microbiota than with the gastric microbiota (Table 3). 


\subsection{Differences in Microbial Composition between Corpus- and Antrum-Dominant Atrophic Gastritis}

A mild degree of atrophy was found in the antrum in 43 subjects and in the body in 36 subjects. Among these subjects, 20 showed a mild degree of atrophy in both the antrum and the body. Neither a moderate nor marked degree of atrophy was found in this study. The relative abundance of $H$. pylori $(p=0.001)$, M. osloensis $(p=0.018)$, P. acnes $(p=0.011)$, and Bacillus sp. $(p=0.004)$ differed between the 23 antrum- and 16 corpus-dominant cases of atrophic gastritis. The median relative abundance of $H$. pylori $(0.62 \%$ vs. $0 \%)$ was significantly higher in the antrum- than in the corpus-dominant gastritis cases. Conversely, M. osloensis ( $0.08 \%$ vs. $0.06 \%)$, P. acnes $(9.20 \%$ vs. $3.20 \%)$, and Bacillus sp. $(1.13 \%$ vs. $0.08 \%)$ were more abundant in the corpus- than in the antrum-dominant gastritis cases. Nevertheless, none of these species showed significant differences in their relative abundance between the 79 specimens with atrophy ( 43 antrum and 36 body) and the 117 specimens without atrophy ( 55 antrum and 62 body).

\section{Discussion}

In the present study, microbial communities in the duodenum showed dissimilarity with those in the antrum and body. The relative abundance of $H$. pylori in the stomach was associated with the most cases of histological and endoscopic gastritis, but only some cases of symptomatic gastritis. Only a weak correlation was observed between symptom scores and H. pylori abundance. Symptom scores showed stronger correlation with the duodenal microbiota than with the gastric microbiota. Therefore, avoiding an overgrowth of $H$. pylori may prevent most histological and endoscopic gastritis, but only some symptomatic gastritis. Furthermore, cases of $H$. pylori-negative gastritis involved abundant $V$. paradoxus and P. gingivalis. Such abundances were correlated with histological gastritis, but not with endoscopic or symptomatic gastritis.

Among the 573 gastric species detected in this study, the strongest correlation was observed between the degree of inflammatory cell infiltration and the relative abundance of H. pylori. V. paradoxus and $P$. gingivalis were also linked to inflammation; however, their correlation coefficient values and relative abundance were not comparable to those of H. pylori. Only H. pylori-dominant dysbiosis showed an extremely high relative abundance of up to $98.4 \%$ with significant changes on endoscopic and histological findings. These findings are additive to those of previous studies that reported microbial changes during $H$. pylori-induced chronic inflammation [5,6,8-12]. In those studies, diversity was decreased in H. pylori-dominant condition and was lower in the body than in the antrum. This supports our study findings that correlations between the combined inflammation score and species abundance of $V$. paradoxus and P. gingivalis are more prominent in the body than in the antrum. The impact of certain species seems to be low in a diverse environment such as that in the antrum.

Endoscopic and histological gastritis were associated with the relative abundance of $H$. pylori and those of P. acnes, P. veronii, Pseudomonas sp., C. rupense, and S. epidermidis. Discrepancy between the species related to histological and endoscopic gastritis was noted for $V$. paradoxus and P. gingivalis. The abundances of $V$. paradoxus, and P. gingivalis were correlated with histological gastritis, but not with endoscopic or symptomatic gastritis. The abnormal endoscopic findings observed in this study are consistent $H$. pylori-related endoscopic findings with an increased risk of gastric cancer. Intestinal metaplasia and gastric corpus atrophy increase the risk of intestinal-type gastric cancer, whereas hypertrophic rugae, diffuse redness, and nodularity increase the risk of diffuse-type gastric cancer $[4,17,18,25-27]$.

P. gingivalis is a well-known periodontal pathogen associated with esophageal cancer [28]; however, it did not correlate with endoscopic findings that require gastric cancer surveillance $[4,17,18]$. This study also showed that the abundance of neither $V$. paradoxus nor $P$. gingivalis was associated with atrophy. Only the abundance of $H$. pylori was associated with antrum-dominant atrophic gastritis. These findings support that $H$ pylori-negative gastritis does not progress to precancerous lesions [29].

In this study, the species associated with symptom scores were mostly inconsistent with those related to histological and endoscopic gastritis. A stronger correlation with the duodenal microbiota than with the gastric microbiota was observed only in symptomatic gastritis. The weak correlation between 
the total PAGI-SYM score and the abundance of H. pylori explains why other factors (i.e., female sex, young age, spicy food intake) are more correlated with symptoms than $H$. pylori [1,30-32]. Because the enteric nervous system (ENS) may be regulated by the inflammatory effects of the microbiota resulting in altered symptom sensitivity or cognitive function [33,34], our findings further suggest that the duodenal microbiota (P. nanceiensis and A. rava) and gastric microbiota (H. pylori, N. elongata, and $C$. segmentosum) may negatively affect ENS modulation via neurogenic inflammatory process. This increases understanding of the symptoms of $H$. pylori-negative subjects.

The present study has limitations. First, PAGI-SYM questionnaires were used instead of Rome criteria, because the validity of the Korean version of the Rome III questionnaires was shown to be low [35]. Nevertheless, we found that the species that were correlated with symptom severity differed from those related to histological or endoscopic gastritis. Second, most of the patients who visited the clinic were female subjects who wanted to be examined by a female gastroenterologist; thus, only 21 of our included 98 subjects were male. Because the composition of the microbiota did not differ between our male and female subjects, we assume that our study findings would not have changed by increasing the number of male subjects. Third, we could not confirm whether S. epidermidis, P. acnes, P. veronii, Pseudomonas sp., and C. rupense play a defensive role in reducing histological and endoscopic gastritis. Even in H. pylori-negative subjects, the degree of inflammatory cell infiltration increased with the relative abundance of pathogens ( $V$. paradoxus and P. gingivalis), and none of the commensals demonstrated statistically significant differences. The beneficial role of commensal bacteria requires further investigation.

\section{Conclusions}

Different correlations of the gastric and duodenal microbiota with histological, endoscopic, and symptomatic gastritis were observed for the first time at the species level. Histological gastritis was associated with the relative abundances of H. pylori, V. paradoxus, and P. gingivalis. H. pylori-negative gastritis was not associated with endoscopic or symptomatic gastritis. H. pylori was the only pathogen associated with endoscopic gastritis, which requires gastric cancer surveillance.

Symptomatic gastritis should be evaluated and managed differently from histological and endoscopic gastritis, because it is more strongly correlated with the duodenal microbiota (P. nanceiensis and A. rava) than the gastric microbiota (H. pylori, N. elongata, and C. segmentosum). Thus, factors other than $H$. pylori infection status should be assessed in cases of symptomatic gastritis.

Supplementary Materials: The following are available online at http:/ /www.mdpi.com/2077-0383/8/3/312/s1, Figure S1: Microscopic findings of the biopsy specimens; Table S1: Species with a relative abundance of $>0.10 \%$ found in the antrum, body, and duodenum; Table S2: Relative abundance of significant species and updated Sydney system scores according to the presence of regular arrangement of collecting venules; Table S3: Differences in PAGI-SYM scores according to endoscopic findings; The FASTQ sequence files are available in the National Center for Biotechnology Information (NCBI) Bioproject under the accession number of PRJNA486978.

Author Contributions: Drafting of the manuscript: H.S.H. and S.-Y.L.; Study concept and design: S.-Y.L.; Data acquisition, analysis, and interpretation: H.S.H., S.-Y.L., and J.-H.K.; Performed the research and contributed essential tools: S.Y.O., H.W.M., H.C., and J.-H.K.; Final approval of the study: all authors

Funding: This study was supported by the Korean National Research Foundation.

Acknowledgments: This study was supported by the Korean National Research Foundation (2016R1D1A1B02008937) to S.-Y.L.

Conflicts of Interest: The authors declare no conflict of interest.

\section{References}

1. Kim, S.E.; Park, H.K.; Kim, N.; Joo, Y.E.; Baik, G.H.; Shin, J.E.; Seo, G.S.; Kim, G.H.; Kim, H.U.; Kim, H.Y.; et al. Prevalence and risk factors of functional dyspepsia: A nationwide multicenter prospective study in Korea. J. Clin. Gastroenterol. 2014, 48, e12-e18. [CrossRef] [PubMed] 
2. Dixon, M.F.; Genta, R.M.; Yardley, J.H.; Correa, P. Classification and grading of gastritis. The updated Sydney System. International Workshop on the Histopathology of Gastritis, Houston 1994. Am. J. Surg. Pathol. 1996, 20, 1161-1181. [CrossRef] [PubMed]

3. Venerito, M.; Varbanova, M.; Röhl, F.W.; Reinhold, D.; Frauenschläger, K.; Jechorek, D.; Weigt, J.; Link, A.; Malfertheiner, P. Oxyntic gastric atrophy in Helicobacter pylori gastritis is distinct from autoimmune gastritis. J. Clin. Pathol. 2016, 69, 677-685. [CrossRef] [PubMed]

4. Sugimoto, M.; Ban, H.; Ichikawa, H.; Sahara, S.; Otsuka, T.; Inatomi, O.; Bamba, S.; Furuta, T.; Andoh, A. Efficacy of the Kyoto classification of gastritis in identifying patients at high risk for gastric cancer. Intern. Med. 2017, 56, 579-586. [CrossRef] [PubMed]

5. $\quad$ Eun, C.S.; Kim, B.K.; Han, D.S.; Kim, S.Y.; Kim, K.M.; Choi, B.Y.; Song, K.S.; Kim, Y.S.; Kim, J.F. Differences in gastric mucosal microbiota profiling in patients with chronic gastritis, intestinal metaplasia, and gastric cancer using pyrosequencing methods. Helicobacter 2014, 19, 407-416. [CrossRef] [PubMed]

6. Thorell, K.; Bengtsson-Palme, J.; Liu, O.H.; Palacios Gonzales, R.V.; Nookaew, I.; Rabeneck, L.; Paszat, L.; Graham, D.Y.; Nielsen, J.; Lundin, S.B.; et al. Analysis of the viable microbiota and Helicobacter pylori transcriptome in gastric infection and early stages of carcinogenesis. Infect. Immun. 2017, 85. [CrossRef] [PubMed]

7. Rugge, M.; Genta, R.M. Staging and grading of chronic gastritis. Hum. Pathol. 2005, 36, 228-233. [CrossRef] [PubMed]

8. Ferreira, R.M.; Pereira-Marques, J.; Pinto-Ribeiro, I.; Costa, J.L.; Carneiro, F.; Machado, J.C.; Figueiredo, C. Gastric microbial community profiling reveals a dysbiotic cancer-associated microbiota. Gut 2018, 67, 226-236. [CrossRef] [PubMed]

9. Li, T.H.; Qin, Y.; Sham, P.C.; Lau, K.S.; Chu, K.M.; Leung, W.K. Alterations in gastric microbiota after H. pylori eradication and in different histological stages of gastric carcinogenesis. Sci. Rep. 2017, 7, 44935. [CrossRef] [PubMed]

10. Aviles-Jimenez, F.; Vazquez-Jimenez, F.; Medrano-Guzman, R.; Mantilla, A.; Torres, J. Stomach microbiota composition varies between patients with non-atrophic gastritis and patients with intestinal type of gastric cancer. Sci. Rep. 2014, 4, 4202. [CrossRef] [PubMed]

11. Yang, I.; Woltemate, S.; Piazuelo, M.B.; Bravo, L.E.; Yepez, M.C.; Romero-Gallo, J.; Delgado, A.G.; Wilson, K.T.; Peek, R.M.; Correa, P.; et al. Different gastric microbiota compositions in two human populations with high and low gastric cancer risk in Colombia. Sci. Rep. 2016, 6, 18594. [CrossRef] [PubMed]

12. Sohn, S.H.; Kim, N.; Jo, H.J.; Kim, J.; Park, J.H.; Nam, R.H.; Seok, Y.J.; Kim, Y.R.; Lee, D.H. Analysis of gastric body microbiota by pyrosequencing: Possible role of bacteria other than Helicobacter pylori. J. Cancer Prev. 2017, 22, 115-125. [CrossRef] [PubMed]

13. Jo, H.J.; Kim, J.; Kim, N.; Park, J.H.; Nam, R.H.; Seok, Y.J.; Kim, Y.R.; Kim, J.S.; Kim, J.M.; Kim, J.M.; et al. Analysis of gastric microbiota by pyrosequencing: Minor role of bacteria other than Helicobacter pylori in the gastric carcinogenesis. Helicobacter 2016, 21, 364-374. [CrossRef] [PubMed]

14. Schulz, C.; Schütte, K.; Koch, N.; Vilchez-Vargas, R.; Wos-Oxley, M.L.; Oxley, A.P.A.; Vital, M.; Malfertheiner, P.; Pieper, D. The active bacterial assemblages of the upper GI tract in individuals with and without Helicobacter infection. Gut 2018, 67, 216-225. [CrossRef] [PubMed]

15. Walker, M.M.; Talley, N.J. The role of duodenal inflammation in functional dyspepsia. J. Clin. Gastroenterol. 2017, 51, 12-18. [CrossRef] [PubMed]

16. Lee, S.Y.; Masaoka, T.; Han, H.S.; Matsuzaki, J.; Hong, M.J.; Fukuhara, S.; Choi, H.S.; Suzuki, H. A prospective study on symptom generation according to spicy food intake and TRPV1 genotypes in functional dyspepsia patients. Neurogastroenterol. Motil. 2016, 28, 1401-1408. [CrossRef] [PubMed]

17. Kato, T.; Yagi, N.; Kamada, T.; Shimbo, T.; Watanabe, H.; Ida, K.; Study Group for Establishing Endoscopic Diagnosis of Chronic Gastritis. Diagnosis of Helicobacter pylori infection in gastric mucosa by endoscopic features: A multicenter prospective study. Dig. Endosc. 2013, 25, 508-518. [CrossRef] [PubMed]

18. Lee, S.Y. Endoscopic gastritis, serum pepsinogen assay, and Helicobacter pylori infection. Korean J. Intern. Med. 2016, 31, 835-844. [CrossRef] [PubMed]

19. Dong, Q.; Xin, Y.; Wang, L.; Meng, X.; Yu, X.; Lu, L.; Xuan, S. Characterization of gastric microbiota in twins. Curr. Microbiol. 2017, 74, 224-229. [CrossRef] [PubMed] 
20. Von Rosenvinge, E.C.; Song, Y.; White, J.R.; Maddox, C.; Blanchard, T.; Fricke, W.F. Immune status, antibiotic medication and $\mathrm{pH}$ are associated with changes in the stomach fluid microbiota. ISME J. 2013, 7, 1354-1366. [CrossRef] [PubMed]

21. Oh, S.Y.; Kim, W.Y.; Hwang, T.S.; Han, H.S.; Lim, S.D.; Kim, W.S. Development of an ammonium sulfate DNA extraction method for obtaining amplifiable DNA in a small number of cells and its application to clinical specimens. Biomed. Res. Int. 2013, 2013, 546727. [CrossRef] [PubMed]

22. Mao, D.P.; Zhou, Q.; Chen, C.Y.; Quan, Z.X. Coverage evaluation of universal bacterial primers using the metagenomic datasets. BMC Microbiol. 2012, 12, 66. [CrossRef] [PubMed]

23. Dubey, A.K.; Uppadhyaya, N.; Nilawe, P.; Chauhan, N.; Kumar, S.; Gupta, U.A.; Bhaduri, A. LogMPIE, pan-India profiling of the human gut microbiome using $16 \mathrm{~S}$ rRNA sequencing. Sci. Data 2018, 5, 180232. [CrossRef] [PubMed]

24. Kim, J.; Kim, N.; Jo, H.J.; Park, J.H.; Nam, R.H.; Seok, Y.J.; Kim, Y.R.; Kim, J.S.; Kim, J.M.; Kim, J.M.; et al. An appropriate cutoff value for determining the colonization of Helicobacter pylori by the pyrosequencing method: Comparison with conventional methods. Helicobacter 2015, 20, 370-380. [CrossRef] [PubMed]

25. Shichijo, S.; Hirata, Y.; Niikura, R.; Hayakawa, Y.; Yamada, A.; Koike, K. Association between gastric cancer and the Kyoto classification of gastritis. J. Gastroenterol. Hepatol. 2017, 32, 1581-1586. [CrossRef] [PubMed]

26. Kamada, T.; Inoue, K.; Haruma, K. Kyoto classification of gastritis. Jpn. J. Helicobacter. Res. 2015, 16, 80-85.

27. Watanabe, K.; Nagata, N.; Nakashima, R.; Furuhata, E.; Shimbo, T.; Kobayakawa, M.; Sakurai, T.; Imbe, K.; Niikura, R.; Yokoi, C.; et al. Predictive findings for Helicobacter pylori-uninfected, -infected and -eradicated gastric mucosa: Validation study. World J. Gastroenterol. 2013, 19, 4374-4379. [CrossRef] [PubMed]

28. Yuan, X.; Liu, Y.; Kong, J.; Gu, B.; Qi, Y.; Wang, X.; Sun, M.; Chen, P.; Sun, W.; Wang, H.; et al. Different frequencies of Porphyromonas gingivalis infection in cancers of the upper digestive tract. Cancer Lett. 2017, 404, 1-7. [CrossRef] [PubMed]

29. Shiota, S.; Thrift, A.P.; Green, L.; Shah, R.; Verstovsek, G.; Rugge, M.; Graham, D.Y.; El-Serag, H.B. Clinical manifestations of Helicobacter pylori-negative gastritis. Clin. Gastroenterol. Hepatol. 2017, 15, 1037-1046. [CrossRef] [PubMed]

30. Yoshioka, T.; Takeshita, E.; Sakata, Y.; Hara, M.; Akutagawa, K.; Sakata, N.; Endo, H.; Ohyama, T.; Matsunaga, K.; Tanaka, Y.; et al. Helicobacter pylori infection status had no influence on upper gastrointestinal symptoms: A cross-sectional analysis of 3005 Japanese subjects without upper gastrointestinal lesions undergoing medical health checkups. Esophagus 2017, 14, 249-253. [CrossRef] [PubMed]

31. Lee, S.P.; Lee, S.Y.; Kim, J.H.; Sung, I.K.; Park, H.S.; Shim, C.S. Factors related to upper gastrointestinal symptom generation in 2275 Helicobacter pylori seroprevalent adults. Dig. Dis. Sci. 2017, 62, 1561-1570. [CrossRef] [PubMed]

32. Kawakubo, H.; Tanaka, Y.; Tsuruoka, N.; Hara, M.; Yamamoto, K.; Hidaka, H.; Sakata, Y.; Shimoda, R.; Iwakiri, R.; Kusano, M.; et al. Upper gastrointestinal symptoms are more frequent in female than male young healthy Japanese volunteers as evaluated by questionnaire. J. Neurogastroenterol. Motil. 2016, 22, 248-253. [CrossRef] [PubMed]

33. Konturek, P.C.; Brzozowski, T.; Konturek, S.J. Stress and the gut: Pathophysiology, clinical consequences, diagnostic approach and treatment options. J. Physiol. Pharmacol. 2011, 62, 591-599. [PubMed]

34. Budzyński, J.; Kłopocka, M. Brain-gut axis in the pathogenesis of Helicobacter pylori infection. World J. Gastroenterol. 2014, 20, 5212-5225. [CrossRef] [PubMed]

35. Ghoshal, U.C.; Gwee, K.A.; Chen, M.; Gong, X.R.; Pratap, N.; Hou, X.; Syam, A.F.; Abdullah, M.; Bak, Y.T.; Choi, M.G.; et al. Development, translation and validation of enhanced Asian Rome III questionnaires for diagnosis of functional bowel diseases in major Asian languages: A Rome foundation-Asian neurogastroenterology and motility association working team report. J. Neurogastroenterol. Motil. 2015, 21, 83-92. [CrossRef] [PubMed]

(C) 2019 by the authors. Licensee MDPI, Basel, Switzerland. This article is an open access article distributed under the terms and conditions of the Creative Commons Attribution (CC BY) license (http:// creativecommons.org/licenses/by/4.0/). 